\title{
A Deadly Fungus: Case of an Unidentified Severe Cryptococcal Meningoencephalitis
}

\author{
Santiago Coste-Sibilia ${ }^{\mathrm{a}, \mathrm{b}}$, Juan J. Nieves-Rivera ${ }^{\mathrm{a}}$
}

\begin{abstract}
A 45-year-old man with no systemic illnesses was found by his neighbor lying unconscious and with recurrent involuntary movements, reason for which he was endotracheally intubated and brought to our institution. Upon evaluation, we found an acutely ill-looking man, with decreased level of consciousness (Glasgow coma scale 11) off sedation, on mechanical ventilator support with adequate oxygenation. Physical exam was remarkable for fever, scalp lacerations with old sutures, generalized rigidity and diaphoresis, with clear lungs and no abnormal heart sounds. Laboratory results showed no leukocytosis or leukopenia, but normocytic normochromic anemia; there were no electrolyte disturbances and negative toxicology screen. Head CT without intravenous contrast showed no findings suggesting bleeding, ischemia or space occupying lesions but reported parenchymal disease, and old skull and maxillofacial fractures. Patient's neighbor referred that he was hospitalized 2 weeks ago at another institution with seizures that apparently caused him a 12 -feet fall at work. Reevaluation showed a positive Kernig and Brudzinski signs. Lumbar puncture was performed. The opening pressure was markedly elevated $\left(>30 \mathrm{~cm} \mathrm{H}_{2} \mathrm{O}\right)$ and cerebrospinal fluid (CSF) analysis was consistent with fungal infection. He was promptly started in empiric antifungal therapy with amphotericin and flucytosine while waiting for CSF culture results. Meanwhile, serial lumbar punctures were performed to decrease intracranial pressure. HIV tests were sent and resulted positive. CSF culture showed Cryptococcus neoformans, and CSF cryptococcal antigen titer was positive in 1:32 dilutions. Diagnosis of severe cryptococcal meningoencephalitis was confirmed. Cryptococcus neoformans is one of the leading opportunistic infections seen in untreated patients with HIV and is uniformly fatal within approximately 2 weeks if untreated. Our patient's prognosis was poor since admission; however, we just cannot stop thinking that maybe his prognosis could have been different if meningitis was even suspected and confirmed during patient's previous hospitalization for seizures 2 weeks ago.
\end{abstract}

Manuscript accepted for publication July 09, 2015

anternal Medicine Residency Program, University of Puerto Rico, University District Hospital, San Juan, Puerto Rico

${ }^{b}$ Corresponding Author: Santiago Coste-Sibilia, University of Puerto Rico Medical Sciences Campus Internal Medicine Department, PO Box 365067, San Juan PR 00936-5067, Puerto Rico. Email: santiago.coste@upr.edu

doi: http://dx.doi.org/10.14740/jmc2194w
Keywords: Cryptococcus; Meningoencephalitis; Seizure; Trauma; Misdiagnosis

\section{Introduction}

Cryptococcus neoformans meningoencephalitis is the most frequently encountered manifestation of cryptococcosis. It is the leading cause of central nervous system (CNS) fungal infections worldwide, and one of the acquired immunodeficiency syndrome (AIDS) defining illnesses in $60-70 \%$ of human immunodeficiency virus (HIV)-infected patients [1]. It is caused by the basidiomycetous, encapsulated yeast Cryptococcus neoformans serotypes A and D [1]. Early diagnosis and treatment of cryptococcal meningoencephalitis is determinant in the outcome of this highly fatal disease, if left untreated [2]. Diagnosis can be challenging, particularly if it presents with non-specific symptomatology. We are presenting a case of a late-diagnosed cryptococcal meningoencephalitis failed to be identified 2 weeks after the initial presentation with seizures de novo.

\section{Case Report}

A 45-year-old man with no systemic illnesses was found by his neighbor lying unconscious and with involuntary movements. He was endotracheally intubated, and brought to our institution for evaluation. Our service was consulted for mechanical ventilator and seizures management. Upon evaluation, we found an acutely ill-looking man, with decreased level of consciousness (Glasgow coma scale: eyes 4, verbal 1, motor 6) off sedation, on mechanical ventilator support with adequate oxygenation by arterial blood gases. Patient was normotense, but febrile, tachycardic, and tachypneic. Physical exam was remarkable for scalp lacerations with old sutures, generalized rigidity and diaphoresis, with clear lungs and no abnormal heart sounds. Laboratory results showed no leukocytosis or leukopenia, but normocytic normochromic anemia; no electrolyte disturbances and negative toxicology screen were found as shown in Table 1. Head CT without intravenous contrast showed no findings suggesting bleeding, ischemia or space occupying lesions but parenchymal disease reported as shown in Figure 1. However, it showed old skull and maxillofacial fractures. Further infor- 
Table 1. Laboratory Values

\begin{tabular}{ll}
\hline Sodium $(\mathrm{mEq} / \mathrm{L})$ & 134 \\
Potassium $(\mathrm{mEq} / \mathrm{L})$ & 4.0 \\
Chloride $(\mathrm{mEq} / \mathrm{L})$ & 99 \\
Bicarbonate $(\mathrm{mEq} / \mathrm{L})$ & 25.8 \\
BUN $(\mathrm{mg} / \mathrm{dL})$ & 8 \\
Creatinine $(\mathrm{mg} / \mathrm{dL})$ & 0.53 \\
Glucose $(\mathrm{mg} / \mathrm{dL})$ & 97 \\
AST $(\mathrm{U} / \mathrm{L})$ & 19 \\
ALT $(\mathrm{U} / \mathrm{L})$ & 29 \\
Total bilirubin $(\mathrm{mg} / \mathrm{dL})$ & 0.5 \\
Calcium mg/dL & 8.7 \\
Lactate $(\mathrm{mEq} / \mathrm{L})$ & 6.5 \\
Alkaline $\mathrm{phosphatase}(\mathrm{U} / \mathrm{L})$ & 121 \\
Albumin $(\mathrm{g} / \mathrm{dL})$ & 3.9 \\
Magnesium $(\mathrm{mEq} / \mathrm{L})$ & 1.94 \\
Phosphorus $(\mathrm{mEq} / \mathrm{L})$ & 4.10 \\
\hline
\end{tabular}

mation from neighbor revealed that he was HIV-positive, and that he was hospitalized 2 weeks ago at another institution for multiple body trauma secondary to a seizures-related 12 -feet fall at work. He was discharged home just 2 days before arriving at our institution. Neurological status at that time is un-

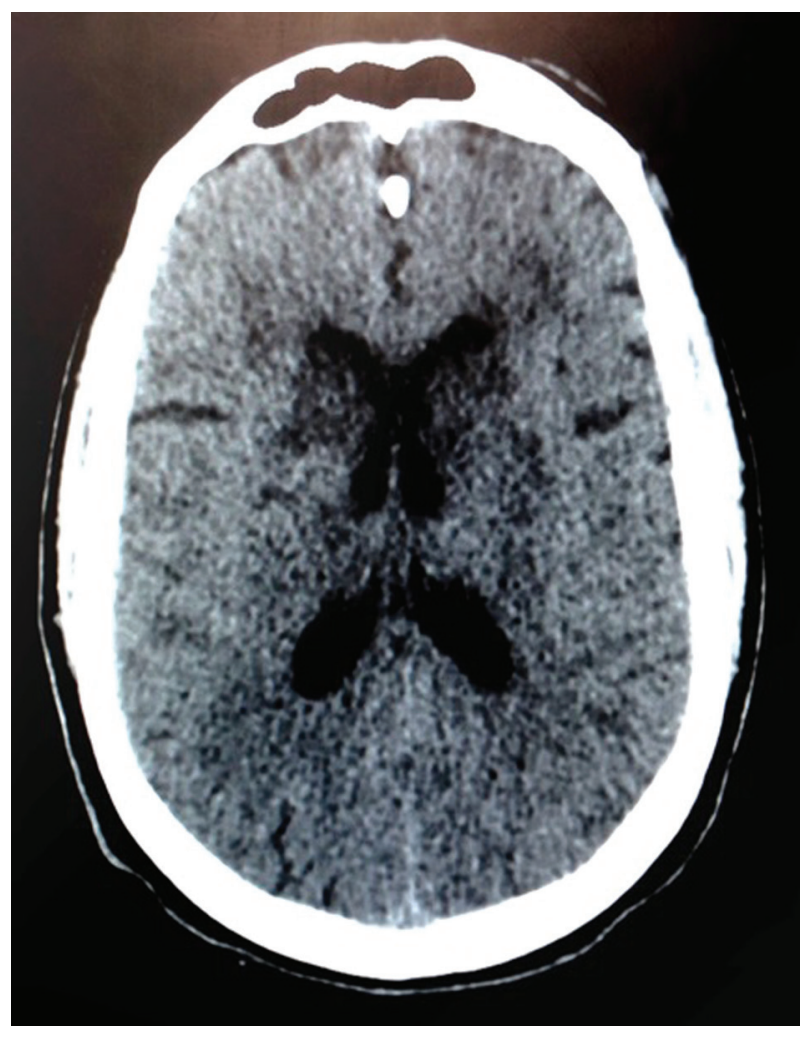

Figure 1. Head CT without contrast.
Table 2. Lumbar Puncture

\begin{tabular}{ll}
\hline Opening pressure & $>30 \mathrm{~cm} \mathrm{H}_{2} \mathrm{O}$ \\
CSF appearance & Cloudy \\
CSF color & Colorless \\
CSF WBC & 23 \\
CSF RBC & 19 \\
CSF protein & 112 \\
Mononuclear (\%) & 65 \\
Polynuclear (\%) & 35 \\
Glucose & $<6$ \\
LDH & 140 \\
India ink & Encapsulated yeast \\
Cryptococcal Ag titer & $1: 32$ \\
Cryptococcal Ag & Positive \\
CSF culture & C. neoformans \\
\hline
\end{tabular}

known. In view of the above, meningitis was highly suspected. Re-evaluation showed positive Kernig and Brudzinski signs. Lumbar puncture was performed and opening pressure was markedly elevated $\left(>30 \mathrm{~cm} \mathrm{H}_{2} \mathrm{O}\right.$ ). Cerebrospinal fluid (CSF) india ink stain revealed encapsulated yeast, and cytology was consistent with fungal infection. He was promptly started in empiric antifungal therapy with amphotericin and flucytosine. HIV tests were sent and came positive. CD4 levels were 88 cell $/ \mu \mathrm{L}$ and HIV RNA quantitative assay showed 38,285 copies/mL. CSF culture grew Cryptococcus neoformans, and CSF cryptococcal antigen titer was positive in 1:32 dilutions as shown in Table 2. Diagnosis of severe cryptococcal meningoencephalitis was confirmed. Serial lumbar punctures were performed to decrease intracranial pressure. Patient was taken to intensive care unit, but even after aggressive antifungal therapy and mechanical ventilator support, no clinical improvement was achieved and patient died just 2 days after arriving.

\section{Discussion}

Seizure alone is a very non-specific clinical presentation of any CNS pathology, particularly, in a patient with an apparent diagnosis of epilepsy who was just brought into the emergency department under unclear circumstances without evident signs of a current infectious process and unknown immunocompromised state. To diagnose cryptococcal meningoencephalitis, one must have high degree of suspicion. In this case, we have a patient who had a seizure 2 weeks before arriving at our institution, resulting in a 12-feet fall and multiple body trauma, including traumatic brain injury. He was hospitalized for 2 weeks receiving supportive care and was discharged home. There was no evidence of antifungal treatment or identification of cryptococcosis as the possible cause of patient's seizure event. Two days after being discharged from the other institution, he was found having another seizure event and was brought to our ER for evaluation. There, we identified that patient really had a severe cryptococcal meningoencephalitis asso- 
ciated to HIV-related immunocompromised state. Antifungal therapy was promptly started; however, he passed away in less than $48 \mathrm{~h}$ after arriving. Cryptococcus neoformans is one of the leading opportunistic infections seen in untreated patients with HIV, and is uniformly fatal within approximately 2 weeks, if left untreated [3]. Our patient's prognosis was poor since admission; however, we just cannot stop thinking that maybe his prognosis could have been different if meningitis was even suspected and confirmed during patient's previous hospitalization for seizures the 2 weeks before.

Disseminated Cryptococcus neoformans infection is a serious opportunistic infection that occurs in patients with untreated AIDS [3]. Although cryptococcal infections begin in the lungs, meningoencephalitis is the most frequently encountered manifestation of cryptococcosis among those with advanced immunosuppression [4]. If not properly identified and managed on time, it becomes highly fatal [4].

\section{Grant Support}

None.

\section{Conflict of Interest}

None.

\section{References}

1. Park BJ, Wannemuehler KA, Marston BJ, Govender N, Pappas PG, Chiller TM. Estimation of the current global burden of cryptococcal meningitis among persons living with HIV/AIDS. AIDS. 2009;23(4):525-530.

2. Jarvis JN, Harrison TS. HIV-associated cryptococcal meningitis. AIDS. 2007;21(16):2119-2129.

3. Robinson PA, Bauer M, Leal MA, Evans SG, Holtom PD, Diamond DA, Leedom JM, et al. Early mycological treatment failure in AIDS-associated cryptococcal meningitis. Clin Infect Dis. 1999;28(1):82-92.

4. Saag MS, Graybill RJ, Larsen RA, Pappas PG, Perfect JR, Powderly WG, Sobel JD, et al. Practice guidelines for the management of cryptococcal disease. Infectious Diseases Society of America. Clin Infect Dis. 2000;30(4):710-718. 